\title{
Research article \\ A study of second to fourth digit ratio in relation to verbal and numerical intelligence among medical students
}

\author{
Grisilda V. B. ${ }^{1}$, Bhat S. ${ }^{2}$, Kavitha L. B. ${ }^{3}$ \\ ${ }^{1}$ Associate Professor, Department of Biochemistry, RAK College of Medical Sciences, RAK Medical and Health Science \\ University, RAK, UAE \\ ${ }^{2}$ Associate Professor, Department of Microbiology, ${ }^{3}$ Associate Professor, Department of Physiology, Melaka Manipal Medical \\ College, Manipal Campus, Manipal Academy of Higher Education, Manipal, Karnataka, India
}

(Received: December $2020 \quad$ Revised: May $2021 \quad$ Accepted: May 2021)

Corresponding author: Sujatha Bhat. Email: sujatha.raja@ manipal.edu

\begin{abstract}
Introduction and Aim: Prenatal androgens are believed to be one of the probable etiological factors influencing intellectual development of an individual. In-utero testosterone exposure has been thought to affect the digit ratio which is the ratio of lengths of index finger and ring finger (2D:4D). In the present work we intended to study the correlation of 2D:4D with numerical and verbal intelligence believed to be variable among genders. The aim of our study was to find the association of 2D:4D with Verbal Intelligence and Numerical Intelligence in a sample of medical students.
\end{abstract}

Materials and Methods: This study was conducted in a sample of medical students. 44 female and 44 male students participated in the study. 2D:4D measurements were made using standard procedure after which the participants completed a questionnaire containing 20 questions each to test numerical and verbal intelligence.

Results: In this study, Males were found to have a lower 2D:4D when compared to females. Individuals with lower 2D:4D in their right-hand were seen to have lower verbal intelligence and higher numerical intelligence thus suggesting that males are born with better numerical intelligence and females with greater verbal intelligence.

Conclusion: Digit ratio can be considered as a valuable indicator of individual differences in terms of intelligence, although the exact role of testosterone on brain to cause variation in these cognitive domains is yet to be clearly understood.

Keywords: Digit ratio; verbal intelligence; numerical intelligence.

\section{INTRODUCTION}

$\mathrm{T}$ he ratio of the lengths of $2^{\text {nd }}$ to $4^{\text {th }}$ digits (2D:4D) is found to be sexually dimorphic. Greater proportion of males has shorter index finger than ring finger compared to that of women $(1,2)$. The sex differences occur at a prenatal age of 13-14 weeks post-conception (3) and is highly dependent on prenatal androgen exposure during the fetal development (4). Both sex steroids, estrogen and androgen have been found to play a dominant role as a deciding factor of morphological sex differences during the early fetal development. The masculinizing and defeminizing influence of low prenatal oestrogens, high prenatal androgens or both produces a low 2D:4D (5). The sexual differentiation of the brain has also been assumed to depend on these steroid hormones.

In humans, low 2D:4D is well correlated to a masculine pattern with higher numerical intelligence/performance(6). Similarly, females with high 2D:4D is well correlated to low/absence of prenatal testosterone which causes feminine pattern with high verbal intelligence $(7,8)$. In a study conducted by Luxen MF and his associate, a positive correlation between verbal intelligence and right hand 2D:4D was reported. Numerical intelligence was seen to be higher in individuals with lower rigt hand 2D:4D (7).

The aim of the present study is to determine relationship between the 2D:4D of an individual and his/her numerical and verbal intelligence, both in male and female students of Private Medical College, Karnataka, India. To the best of our knowledge no research has been carried out so far to evaluate the relation between 2D:4D and verbal and numerical intelligence in medical students.

\section{MATERIALS AND METHODS}

A sample of 88 (44 male and 44 female) student volunteers was studied at a Private Medical College in Karnataka, India. Institutional ethical clearance was obtained to carry out this work (IEC 293/2015). Within this sample, students from MBBS and BDS courses were included. The lengths of the index finger (second digit) and the ring finger (fourth digit) at the palm-side were measured with a measurement scale. We took direct measurements of the hands, not of photocopies of the hands. Right hand was chosen for the study because the right hand was found to show more significant results (12). 2D:4D was calculated by dividing the length of the index finger by the length of the ring finger. After the measurements were made, a 
questionnaire containing 20 numerical and 20 verbal questions were distributed to all the 88 student volunteers. Informed consent was taken before they were asked to respond to the questionnaire. The students were then divided in to five categories based on the scores, the details of which are shown in table 1.

\section{Table 1: Categories to which students fall based on} the scores

\begin{tabular}{|c|c|}
\hline Category & Scores \\
\hline Genius & 10 \\
\hline Gifted & $8-9$ \\
\hline Above average & $4-7$ \\
\hline Below average & $2-3$ \\
\hline Poor & $0-1$ \\
\hline
\end{tabular}

\section{RESULTS}

Fig. 1 presents sex differences in 2D:4D ratios for all participannts. Among 15 students with 2D:4D lower than $1,66 \%$ were males. We also observed that, out of 36 students with 2D:4D ratio of more than one, $61 \%$ were females. It is clear from the figure that, the digit ratio is a dimorphic trait with respect to gender.

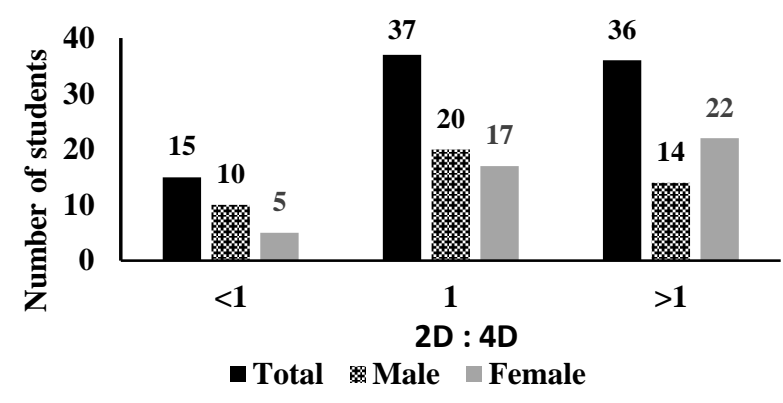

Fig. 1: 2D: 4D distribution in male and female students

Fig. 2 presents finding on numerical intelligence among male and female participants. $16 \%$ of males were gifted and $52 \%$ of males showed above average numerical intelligence. Very small percentage of male participants $(7 \%)$ in our study showed poor numerical intelligence. In contrast, among female participants, only $50 \%$ showed above average numerical intelligence, $4.5 \%$ were gifted. Overall, $68 \%$ of males showed higher numerical skills as compared to that of females where it was found to be $54 \%$. Thus numerical intelligence level was observed to be higher in males than in females.

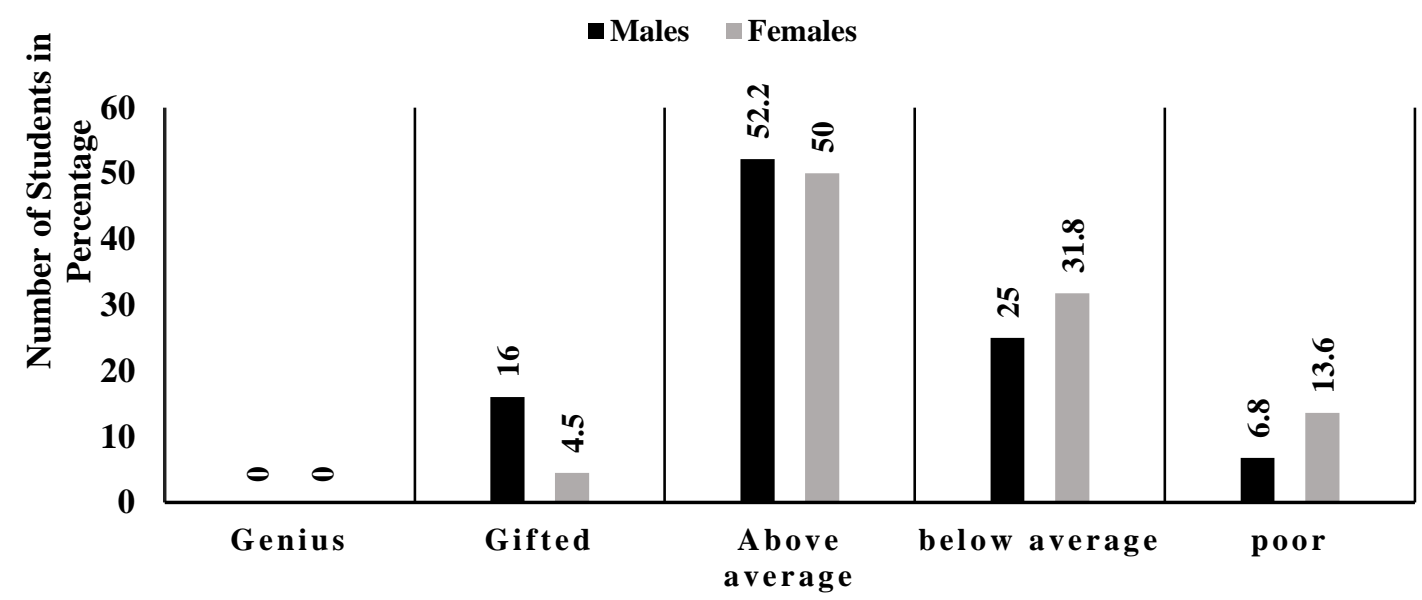

Fig. 2: Percentage of males and females with numerical intelligence

Fig. 3 shows the percentage of males and females with verbal intelligence. We have observed that $16 \%$ of males were gifted, $11 \%$ were genius and $50 \%$ showed above average intelligence. Among females, $18 \%$ were gifted, $4.5 \%$ were genius and $45 \%$ showed above average intelligence. Our results show that males score better than females in the verbal intelligence.

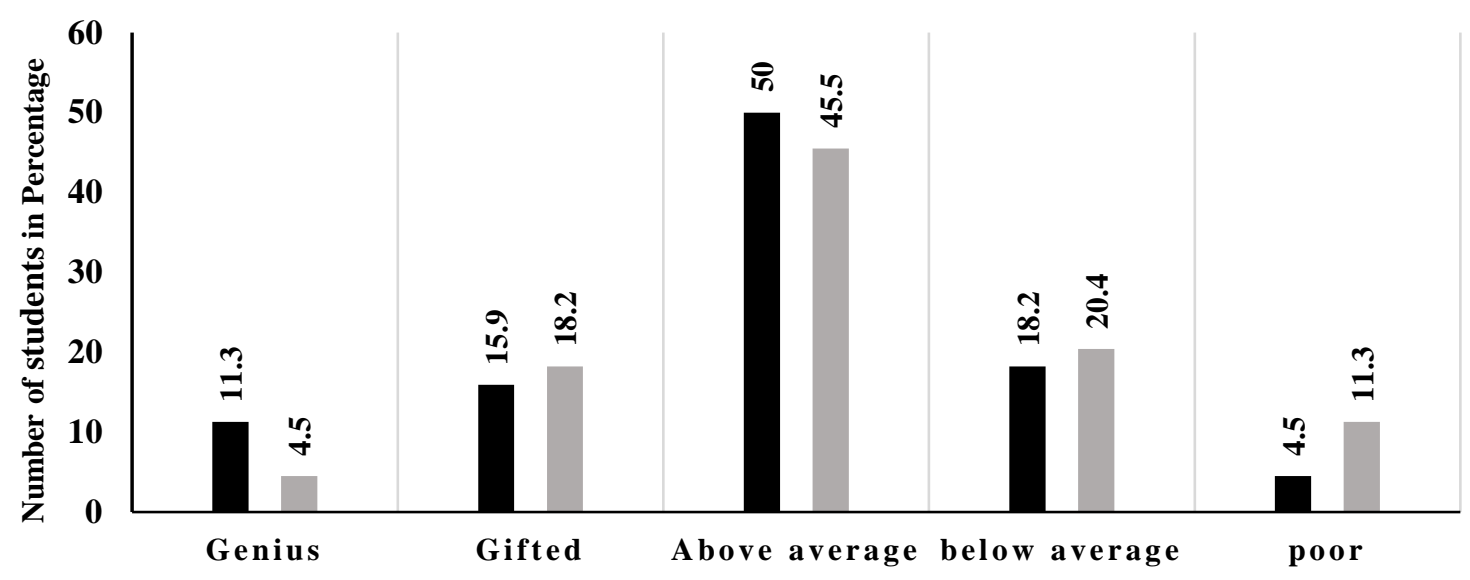

Fig. 3: Percentage of males and females with verbal intelligence 


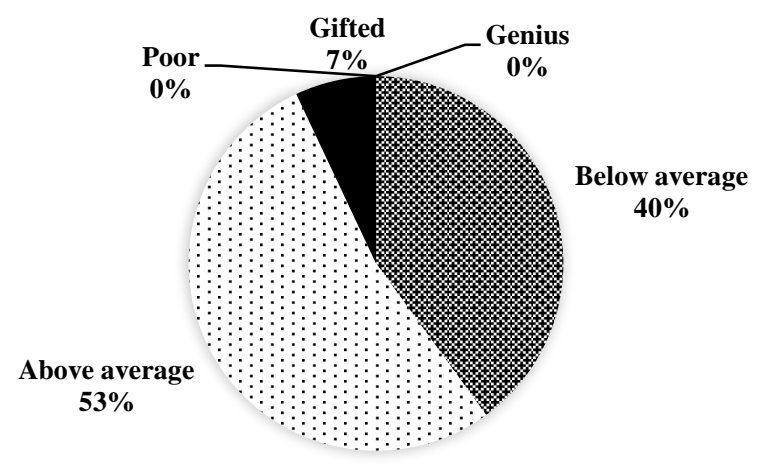

Fig. 4: Numerical intelligence among the students with 2D:4D ratio $<1$

Since in our sudy groups, male students were seen to score better both in assessment of numerical skills and in verbal intelligence when compared to females, we considered the students with the $2 \mathrm{D}: 4 \mathrm{D}$ ratio $<1$ to assess numerical skills and students with 2D:4D ratio $>1$ to check the verbal intelligence, irrespective of gender. Fig. 4 shows the numerical Intelligence among the students with $2 \mathrm{D}: 4 \mathrm{D}$ ratio $<1$. It is observed that, about $53 \%$ of the students showed above average, $7 \%$ of them were gifted and $40 \%$ of them were below average numerical intelligence.
However there were no poor or genius students. When the verbal intelligence were compared among the students with 2D:4D ratio > 1 (Fig. 5), we observed that about $38 \%$ of them were gifted with verbal intelligence and $37 \%$ of them were showing above average intelligence. Only $3 \%$ of the students showed poor intelligence. In this study, although,males seem to outperform the females with respect to both numerical and verbal skills, digit ratio showed negative correlation with numerical intelligence and positive correlation with verbal intelligence.

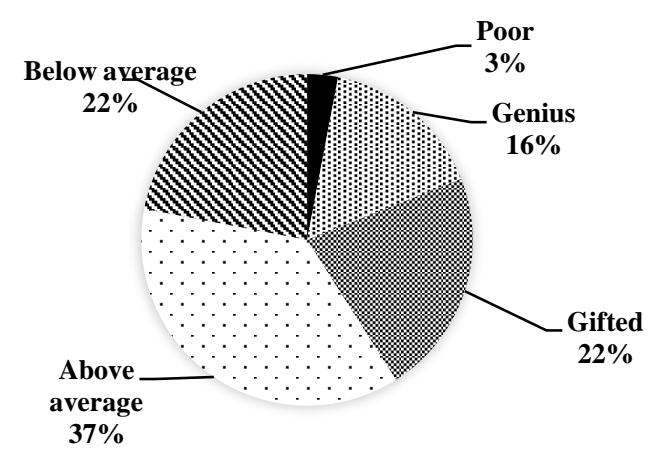

Fig. 5: Verbal intelligence among the students with 2D:4D ratio > 1

\section{DISCUSSION}

The current study found that males have lower 2D:4D, and this finding is in line with many other earlier findings $(1,4)$. It is a well established hypothesis that digit ratios are associated with either higher prenatal testosterone levels or greater sensitivity to androgens, or both (12). In this study we also aimed at finding the correlation between 2D:4D and numerical intelligence in a population of medical students. Various studies have revealed that males have an advantage over females with respect to numerical skills. Royer et al (9) has made an extensive review in this regard and have put forth the report that gender difference do exist with respect to this domain, and also that, a number of variables like the mathematical content of the tests and age and overall ability of the individuals assessed effects the magnitude of the sex difference in mathematical abilities. The effect of 2D:4D on numerical skill has not been studied in depth. Along with the association of gender with different levels of numerical ability we also found that, among the participants with lower than one digit ratio,larger percentage showed above average numerical intelligence.

Verbal skills represent ones ability to use language, in terms of grammar, spellings, vocabulary, verbal analogies and oral comprehension (10). Substantial number of studies related to gender effect on verbal skills have reported that females have an advantage, over males, in performance of verbal tasks (11). In this study, when verbal intelligence was assessed with respect to gender, we obtained a result that is contrary to this common understanding that, females are gifted with better verbal intelligence. Verbal intelligence, like the numerical skills, has been shown to vary with age and the general IQ levels (12). However recent literatures have suggested that the existence of gender differences for verbal skills is not true for all components of this trait. Males have shown to perform better in verbal analogies, whereas females are favoured with grammar, spellings, reading and writing (13). Moreover, few studies have found no significant 
sex difference in verbal reasoning (14). We also analysed the correlation between 2D:4D and verbal skill, wherein we assessed the percentage of each category under this trait among students with $2 \mathrm{D}: 4 \mathrm{D}>1$, without considering the gender. Among the students with higher than 1 digit ratio, a major percentage exhibited above average verbal skills, wheras a very small percentage fell into the below average and poor category. Similar result was reported in a study conducted in a Dutch sample of 44 men and 37 women in 2005 . They found a positive correlation between digit ratio and verbal intelligence (7).

While the basis for origin of intelligence remains uncertain, the concept of cerebral dominance states that exposure of fetus to higher concentrations of testosterone in utero augments the development of right brain giving rise to higher intelligence (15). It was discussed in a review on monozygotic male twin that high level of testosterone in prenatal fetus is associated with higher level of intellect (16). Although the influence of testosterone has been widely studied, recently it is being argued that testosterone is one of few biological factors affecting the organization of developing brain and also the modulation of cognitive functions (17).

Even though the sample size of our study was small, our research yielded more conclusive results on the influence of testosterone as a deciding factor for the development of masculine/feminine traits in an individual.

\section{CONCLUSION}

We conclude that the digit ratio is sexually dimorphic and 2D:4D digit ratio can be compared to person's numerical and verbal intelligence. This may be the result of the prenatal testosterone which influences the brain during early development to acquire either masculine or feminine traits. The persons with high 2D:4D ratio express more feminine features and lower 2D:4D ratio express masculine features.

\section{CONFLICT OF INTEREST}

Authors declare no conflict of interest.

\section{REFERENCES}

1. Manning, J. T., Scutt, D., Wilson, J., Lewis-Jones, D. I. The ratio of 2nd to 4th digit length: a predictor of sperm numbers and concentrations of testosterone, luteinizing hormone and oestrogen. Hum Reprod. 1998; 13(11): 3000-3004.

2. Baker, F. Anthropological notes on the human hand. American Anthropologist. 1888; 1(1): 51-76.

3. Garn, S. M., Burdi, A. R., Babler, W. J., Stinson, S. Early prenatal attainment of adult metacarpal-phalangeal rankings and proportions. American Journal of Physical Anthropology 1975; 43(3): 327-332.

4. McIntyre, M. H. The use of digit ratios as markers for perinatal androgen action. Reproductive Biology and Endocrinology. 2006; 4(1): 10.

5. Brown, W. M., Hines, M. Breedlove, S. M. Masculinized finger length patterns in human males and females with congenital adrenal hyperplasia. Hormones and behavior 2002; 42(4): 380-386.
6. Fink, B., Brookes, H., Neave, N., Manning, J. T., Geary, D. C. Second to fourth digit ratio and numerical competence in children. Brain and cognition. 2006; 61(2): 211-218.

7. Luxen, M. F., Buunk, B. P. Second-to-fourth digit ratio related to verbal and numerical intelligence and the Big Five. Personality and Individual Differences 2005; 39(5): 959-966.

8. Fink, B., Manning, J. T., Neave, N. Second to fourth digit ratio and the 'big five'personality factors. Personality and Individual Differences. 2004; 37(3): 495-503.

9. Royer, J. M., Tronsky, L. N., Chan, Y., Jackson, S. J., Marchant, H, 3rd. Math-fact retrieval as the cognitive mechanism underlying gender differences in math test performance. Contemporary educational psychology. 1999; 24(3): 181-266.

10. Halpern, D. F. Sex differences in cognitive abilities: Psychology press. 2013.

11. Toivainen, T., Papageorgiou, K. A., Tosto, M. G., Kovas, Y. Sex differences in non-verbal and verbal abilities in childhood and adolescence. Intelligence 2017; 64: 81-88.

12. Vogel, S. A. Gender differences in intelligence, language, visual-motor abilities, and academic achievement in students with learning disabilities: A review of the literature. Journal of Learning Disabilities 1990; 23(1): 44-52.

13. Stoet, G., Geary, D. C. Sex differences in mathematics and reading achievement are inversely related: Within-and across-nation assessment of 10 years of PISA data. PloS one. 2013; 8(3): e57988

14. Lynn, R., Allik, J., Pullmann, H., Liadra, K. Sex differences on the progressive matrices among adolescents: Some data from Estonia. Personality and Individual Differences. 2004; 36(6): 1249-1255.

15. Geschwind, N., Galaburda, A. M. Cerebral lateralization: Biological mechanisms, associations, and pathology: II. A hypothesis and a program for research. Archives of Neurology. 1985; 42(6): 521-552.

16. Fingelkurts, A. A., Fingelkurts, A. A. Exploring giftedness. Advances in psychology research. 2002; 9: 137-155.

17. Auyeung, B., Lombardo, M. V., Baron-Cohen, S. Prenatal and postnatal hormone effects on the human brain and cognition. Pflügers Archiv-European Journal of Physiology. 2013; 465(5): 557-571. 